\title{
Fault-Tolerant Approximate Shortest-Path Trees
}

\author{
Davide Bilò • Luciano Gualà . \\ Stefano Leucci · Guido Proietti
}

\begin{abstract}
The resiliency of a network is its ability to remain effectively functioning also when any of its nodes or links fails. However, to reduce operational and set-up costs, a network should be small in size, and this conflicts with the requirement of being resilient. In this paper we address this trade-off for the prominent case of the broadcasting routing scheme, and we build efficient (i.e., sparse and fast) faulttolerant approximate shortest-path trees, for both the edge and vertex single-failure case. In particular, for an $n$-vertex non-negatively weighted graph, and for any constant $\varepsilon>0$, we design two structures of size $O\left(\frac{n \log n}{\varepsilon^{2}}\right)$ which guarantee $(1+\varepsilon)$ stretched paths from the selected source also in the presence of an edge/vertex failure. This favorably compares with the currently best known solutions, which are for the edge-failure case of size $O(n)$ and stretch factor 3, and for the vertexfailure case of size $O(n \log n)$ and stretch factor 3. Moreover, we also focus on the unweighted case, and we prove that an ordinary spanner can be slightly augmented in order to build efficient fault-tolerant approximate breadth-first-search trees.
\end{abstract}

Keywords Shortest-path Trees, Fault-tolerant Structures, Approximate Distances

A preliminary version of this paper appeared on the Proceedings of the 22nd European Symposium on Algorithms (ESA'14), September 8-10, 2014, Wroclaw, Poland, Vol. 8737 of Lecture Notes in Computer Science, Springer, pp. 137-148. This work was partially supported by the Research Grant PRIN 2010 "ARS TechnoMedia", funded by the Italian Ministry of Education, University, and Research.

Davide Bilò

Dipartimento di Scienze Umanistiche e Sociali, Università di Sassari, Italy.

E-mail: davide.bilo@uniss.it

Luciano Gualà

Dipartimento di Ingegneria dell'Impresa, Università di Roma "Tor Vergata", Italy.

E-mail: guala@mat.uniroma2.it

Stefano Leucci

Dipartimento di Informatica, "Sapienza" Università di Roma, Italy.

E-mail: leucci@di.uniroma1.it

Guido Proietti

Dipartimento di Ingegneria e Scienze dell'Informazione e Matematica, Università degli Studi dell'Aquila, Italy and Istituto di Analisi dei Sistemi ed Informatica, CNR, Roma, Italy.

E-mail: guido.proietti@univaq.it 


\section{Introduction}

Broadcasting a message from a source node to every other node of a network is one of the most basic communication primitives. Since this operation should be performed by making use of a both sparse and fast infrastructure, the natural solution is to root at the source node a shortest-path tree (SPT) of the underlying graph. However, the SPT, as any tree-based network topology, is highly sensitive to a link/node malfunctioning, which will unavoidably cause the disconnection of a subset of nodes from the source.

To be readily prepared to react to any possible (transient) failure in a SPT, one has then to enrich the tree by adding to it a set of edges selected from the underlying graph, in order to obtain a subgraph that approximately preserves the distance from the source vertex even when a single component (i.e., edge or vertex) fails. More formally, if $s$ denotes a distinguished source vertex of an undirected graph $G=(V(G), E(G))$ with non-negative real weights on its edges, we say that a spanning subgraph $H$ of $G$ is an Edge-fault-tolerant $\alpha$-Approximate SPT (in short, $\alpha$-EASPT), with $\alpha>1$, if it satisfies the following condition: For each edge $e \in E(G)$, all the distances from $s$ in the subgraph $H-e=(V(H), E(H) \backslash\{e\})$ are at most $\alpha$ times longer than the corresponding distances in $G-e$. When vertex failures are considered, then the EASPT is correspondingly called VASPT. Ideally we would like a E/VASPT to have both a low stretch $\alpha$ and a small size, measured as the number $|E(H)|$ of edges in $H$. The case in which $\alpha=1$ corresponds to requiring all the post-failure distances in $H-e$ to match the distances in $G-e$, i.e., $H$ must contain a SPT (from $s$ ) of $G-e$ for every $e \in E(G)$. However, in this case, it is easy to see that $\Omega\left(n^{2}\right)$ edges might be required, as shown in Figure 1 .

The aim of this paper is to show that, as soon as we allow for approximate distances, we can obtain an almost optimal stretch-size tradeoff for E/VASPTs.

\subsection{Related work}

A problem that is very closely related to the design of a E/VASPT is that of computing a single-source distance sensitivity oracle (SDSO). Designing an efficient SDSO means to compute, with a low preprocessing time, a compact data structure which is able to quickly return a (possibly approximate) distance between a source vertex $s$ and any other vertex of the graph, following a component failure. Notice that any E/VASPT $H$ also implies the existence of a (trivial) SDSO having the same size, the same stretch, and a query time of $O(|E(H)|+n \log n)$ : this SDSO is obtained by storing the whole graph $H$ and by running Dijkstra's algorithm from $s$ on the surviving graph to answer queries.

In 5 the authors compute in $O\left(m \log n+n^{2} \log n\right)$ time a SDSO of size $O(n \log n)$, which reports, in constant time per query, 3-stretched distances following the failure of a single vertex. Such an oracle is also path-reporting, i.e., it is able to return the path associated with a distance query by paying an additional time which is proportional to the number of edges it contains. A closer inspection of this result shows that this SDSO is actually obtained through the computation of a 3-VASPT of size $O(n \log n)$. Regarding single edge failures, in [20] are (implicitly) provided (i) a path-reporting SDSO having stretch 3 , size $O(n)$, and constant query time, and 


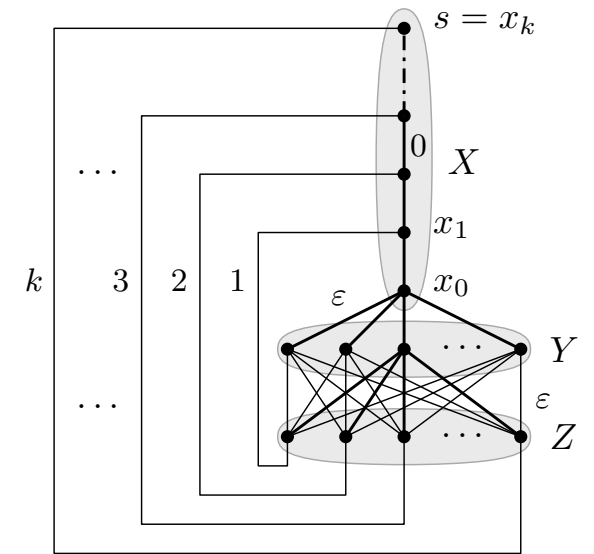

Fig. 1 A weighted graph $G$ on $n$ vertices and $\Theta\left(n^{2}\right)$ edges such that an edge-fault-tolerant SPT $H$ of $G$ is $G$ itself. The vertices are partitioned into three sets $X=\left\{x_{0}, \ldots, x_{k}=s\right\}$, $Y=\left\{y_{1}, \ldots, x_{k}\right\}$, and $Z=\left\{z_{1}, \ldots, z_{k}\right\}$. Vertices in $X$ are connected by a path whose edges have weight 0 , there is a star centered in $x_{0}$ whose leaves are the vertices in $Y$, and the sets $Y$ and $Z$ induce a complete bipartite graph. All edge weights of the star and the complete bipartite graph are equal to $\varepsilon$ with $0<\varepsilon<\frac{1}{2}$, while, for $0<i<k$, there is an edge of cost $i$ between $x_{i}$ and $z_{i}$. A SPT of $G$ from $s$ is shown with bold edges. If any edge $e=\left(x_{i}, x_{i+1}\right)$ fails the new SPT of $G-e$ must include all the edges connecting $z_{i}$ to the vertices in $Y$.

(ii) a corresponding 3 -EABF ${ }^{1}$ containing $2(n-1)$ edges. Very recently, in [8], the authors show how to build a (non path-reporting) SDSO having stretch $1+\varepsilon$, size $O(n \delta)$ and query time $O(\delta \log n)$, where $\delta=\varepsilon^{-1} \log \varepsilon^{-1}$, which can be improved to $O(1)$ for the special case $\varepsilon=1$.

If we focus on unweighted graphs and we insist on preserving exact distances (i.e., stretch equal to 1) then, in 24, the authors provide a 1-E/VABFS of size $O(n \cdot \min \{\operatorname{ecc}(s), \sqrt{n}\})$, where $\operatorname{ecc}(s)$ denotes the eccentricity of the source $s$ in $G$. In the same paper, the authors also exhibit a corresponding lower bound of $\Omega\left(n^{3 / 2}\right)$ for the size of such a structure (in fact, the construction provided in Figure 1 is obtained by elaborating such lower bound). In [5] the authors focus on the vertex-failure case and, for any $\varepsilon>0$, they compute in $O(m \sqrt{n / \varepsilon})$ time a path-reporting SDSO of size $O\left(\frac{n}{\varepsilon^{3}}+n \log n\right)$, stretch $(1+\varepsilon)$, and having constant query time. Once again, this SDSO is obtained through the construction of a $(1+\varepsilon)$ VABFS of size $O\left(\frac{n}{\varepsilon^{3}}+n \log n\right)$. Actually, we point out that the latter structure can be easily sparsified so as to obtain, for any $\varepsilon>0$, a $(1+\varepsilon)$-EABFS of size $O\left(\frac{n}{\varepsilon^{3}}\right)$ : indeed, its $O(n \log n)$-size term is associated with an auxiliary substructure that, for the case of edge failures, can be made of linear size. This result is of independent interest, since it qualifies itself as the best current solution for the EABFS problem. In 25 the authors present, among other results, a 3-EABFS having at most $4 n$ edges. Interestingly, this was the first explicit construction for the problem, but two (better) implicit solutions were already available in the literature: the first one is the just mentioned structure which can be derived from the results presented in [5], while the second one is the 3-EASPT of size at most $2 n$ (and then, a fortiori,

1 We use the notation E/VABFS instead of E/VASPT to stress the fact that we are dealing with unweighted graphs. 
a 3-EABFS of the same size) of [20] that can be easily obtained as a by-product of the results given therein (we will discuss this point in more detail later).

\subsection{Our results}

Our main result is a polynomial time construction 2 of a $(1+\varepsilon)$-E/VASPT of size $O\left(\frac{n \log n}{\varepsilon^{2}}\right)$, for any $\varepsilon>0$. These two structures substantially improve the stretch of the 3-EASPT of linear size implicitly given in [20], and that of the 3-VASPT of size $O(n \log n)$ given in [5], respectively, while essentially using the same number of edges (up to a logarithmic factor in the former case). To obtain our results, we perform a careful selection of edges that will be added to an initial SPT. The somewhat surprising outcome of our approach is that if we accept to have slightly stretched fault-tolerant paths, then we can drastically reduce the $\Theta\left(n^{2}\right)$ size of the structure that we would have to pay for having fault-tolerant shortest paths! Actually, the analysis of the stretch factor and of the structures' size induced by our algorithms is quite involved. Thus, for clarity of presentation, we give our result in two steps: first, we show an approach to build a $(1+\varepsilon)$-EASPT of size $O\left(\frac{n \log n}{\varepsilon^{2}}\right)$, then we outline how this approach can be extended to the vertex-failure case.

We also focus on the unweighted case, and we exhibit an interesting connection between a fault-tolerant approximate BFS and an $(\alpha, \beta)$-spanner. An $(\alpha, \beta)$-spanner of a graph $G$ is a spanning subgraph $H$ of $G$ such that all the node-to-node distances in $H$ are stretched by at most a multiplicative factor of $\alpha$ plus an additive term of $\beta$ w.r.t. the corresponding distances in $G$. If such a condition holds even after an edge/vertex is deleted from both $G$ and $H$, then $H$ is an edge/vertex-fault-tolerant $(\alpha, \beta)$-spanner. Moreover, if the guarantee on the stretch only holds for distances from vertices in a subset $S \subseteq V(G)$, then the spanner is said to be sourcewise. We show how a $(\alpha, \beta)$-spanner of size $\sigma=\sigma(n, m)$ can be used to build in polynomial time a sourcewise edge-fault-tolerant (resp. vertex-fault-tolerant) $(\alpha, \beta)$-spanner of size $O(\sigma+|S| \cdot n)$ (resp., $O(\sigma+|S| \cdot n \log n))$. This result has three main consequences. First of all notice that when $|S|=1$, a sourcewise edge/vertex-fault-tolerant $(\alpha, 0)$ spanner is exactly an $\alpha$-E/VABFS. As a consequence, for relevant values of $\alpha$ and $\beta$ (e.g., when they are constant) the E/VABFS problem is easier than the corresponding (non fault-tolerant) spanner problem, and we regard this as an interesting hardness characterization 3 A second consequence, is that this bridge between the two problems allows to build the sparsest $(1, \beta)$-VABFS structures known so far, by making use of the vast literature on additive $(1, \beta)$-spanners. More precisely, the $(1,4)$-spanner of size $\widetilde{O}\left(n^{\frac{7}{5}}\right)$ given in [11], and the $(1,6)$-spanner of size $O\left(n^{\frac{4}{3}}\right)$ given in [4, can be used to build corresponding VABFS structures. As a last consequence of our result, we are able to: (i) sparsify, for $|S|=\widetilde{\omega}\left(n^{\frac{1}{15}}\right)$, the sourcewise edgefault-tolerant $(1,4)$-spanner of size $O\left(|S| \cdot n^{\frac{4}{3}}\right)$ given in 25 by reducing its size to $\widetilde{O}\left(n^{\frac{7}{5}}+|S| n\right)$; and (ii) reduce the stretch of the sourcewise vertex-fault-tolerant $(1,8)$-spanner of size $\widetilde{O}\left(n^{\frac{4}{3}}\right)$ given in 21] to $(1,6)$, for $|S|=\widetilde{O}\left(n^{\frac{1}{3}}\right)$ (see Section 6 for the exact bounds of the obtained spanners).

\footnotetext{
2 We do not insist on the time efficiency in building our structures, since the focus of our paper, consistently with the literature, is on the trade-off between their size and their stretch factor.

3 For constant values of $\alpha$ and $\beta$, the size of an $(\alpha, \beta)$-spanner is $\omega(n \log n)$ and hence the additive terms in the size of our E/VABFS are dominated by $\sigma$.
} 
1.3 Other related results

Additive EABFS structures. In addition to the already cited results, in 25] the authors also consider $(\alpha, \beta)$-EABFS, i.e., edge-fault-tolerant structures for which the length of a path is stretched by at most a factor of $\alpha$ plus an additive term of $\beta$. In particular, they prove that $(1,3)$-EABFS structures admit a lower bound of $\Omega\left(n^{5 / 4}\right)$ edges, thus showing an interesting dichotomy between multiplicative and additive stretches, i.e., the fact that additive stretches require super-linear size. Moreover, they construct a $(1,4)$-EABFS of size $O\left(n^{4 / 3}\right)$.

Sourcewise E/VABFS structures. In 24, the same authors extend the already cited 1E/VABFS of size $O\left(n^{\frac{3}{2}}\right)$ to the sourcewise case, i.e., that in which the structure incorporates an edge-fault-tolerant BFS rooted at each vertex of a set $S \subseteq V(G)$. Here, they show the existence of a solution of size $O\left(\sqrt{|S|} \cdot n^{3 / 2}\right)$, which is tight. Moreover, they also consider the optimization problem of constructing a minimum-size sourcewise 1-E/VABFS, and they provide a corresponding tight $O(\log n)$-approximation algorithm.

Multiple edge failures. Regarding multiple edge failures, Parter in 22 presented a 2-edge-fault-tolerant exact BFS having $O\left(n^{5 / 3}\right)$ edges, which is tight, while in [25] it is shown the existence of a $(3(f+1),(f+1) \log n)$-EABFS of size $O(f n)$ for any number $f=O(1)$ of failed edges. This latter result has been improved in 9 where the authors prove the existence of a $(2|F|+1)$-EASPT of size $O(f n)$ which tolerates the failure of any set $F$ of edges of size at most $f$. This structure can be converted into a corresponding SDSO having the same size, and with query time $O\left(|F|^{2} \log ^{2} n\right)$. Moreover, if one is willing to use $O\left(m \log ^{2} n\right)$ space, such an oracle is also able to handle any number of edge failures (i.e., up to $m$ ). In [15], the special case of shortest-path failures was considered, where the set of failing edges $F$ is supposed to form a source-leaf subpath in a given SPT of $G$. In particular, for the case $|F|=2$, they give an SDSO achieving stretch 3 , size $O(n \log n)$, and constant query time.

Directed graphs. For single-source distances on directed graphs with integer positive edge weights bounded by $M$, in [19] it is shown how to build efficiently in $\widetilde{O}\left(M n^{\omega}\right)$ time, where $\omega<2.373$ denotes the matrix multiplication exponent, a randomized edge-fault-tolerant SDSO of size $\Theta\left(n^{2}\right)$ returning in $O(1)$ time distances from the source which are exact w.h.p.

Fault-tolerant spanners. Another setting which is very close in spirit to ours is that of fault-tolerant spanners. In [12, for weighted graphs and any integer $k \geq 1$, the authors present a $(2 k-1,0)$-spanner resilient to $f$ vertex (resp., edge) failures of size $O\left(f^{2} k^{f+1} n^{1+1 / k} \log ^{1-1 / k} n\right)$ (resp., $\left.O\left(f n^{1+1 / k}\right)\right)$. This was later improved through a randomized construction in [16]. For a comparison, the sparsest known $(2 k-1)$-multiplicative ordinary spanner has size $O\left(n^{1+1 / k}\right)[1$, and this is believed to be asymptotically tight due to the long-standing girth conjecture of Erdös [18. Finally, we mention that in [3] it was introduced the resembling concept of resilient spanners, i.e., spanners such that whenever any edge in $G$ fails, then the relative 
distance increases in the spanner are very close to those in $G$, and it was shown how to build a resilient spanner by augmenting an ordinary spanner.

Concerning unweighted graphs, it makes instead sense to study fault-tolerant additive spanners. In particular, Braunshvig et al. [10] proposed the following general approach to build an additive spanner tolerating up to $f$ edge failures: Let $A$ be an $f$-edge-fault-tolerant $(\alpha, 0)$-spanner, and let $B$ be an ordinary $(1, \beta)$ spanner. Then $H=A \cup B$ is an $f$-edge-fault-tolerant $(1,2 f(2 \beta+\alpha-1)+\beta)$ spanner. Recently, in [7] the corresponding analysis has been refined yielding a better additive bound of $2 f(\beta+\alpha-1)+\beta$, and, more in general, improved faulttolerant additive spanners have been presented. Also very close to our present work are the (non-fault-tolerant) sourcewise spanners (which, again, approximately preserves all distances from a given set $S \subseteq V$ of sources). In that respect, in [14 the authors give, for any $k>1$, a structure with additive stretch $2 k$ and size $O\left(n^{1+1 /(2 k+1)}(k|S|)^{k /(2 k+1)}\right)$, which in particular for $k=\log n$ returns a structure with additive stretch $2 \log n$ and size $O(n \sqrt{|S| \log n})$. To the best of our knowledge, no results are instead known for the weighted case.

Further related works. For recent achievements on all-to-all distance sensitivity oracles, we refer the reader to [5,6, 13, 17, while for other results on single-edge/vertex failures spanners/oracles on unweighted graphs, we finally refer the reader to [2,5, 21,23 .

\subsection{Paper organization}

The paper is organized as follows: in Section 2 we introduce the notation that will be used throughout the paper; in Section 3 we revisit one of the swap procedures presented in 20] to formally prove that it can be used to build a simple 3-EASPT; in Section 4 and 5 we present our main results, namely a $(1+\varepsilon)$-EASPT and a $(1+\varepsilon)$-VASPT, respectively; in Section 6 we focus on unweighted graphs, and we show the connection between an E/VABFS and an $(\alpha, \beta)$-spanner; finally, in Section 7 we conclude the paper by outlining few directions for future research.

\section{Notation}

We start by introducing our notation. For the sake of brevity, we give it for the case of edge failures, but it can be naturally extended to the node failure case.

Given a non-negatively real weighted, undirected graph $G$, we will denote by $w_{G}(e)$ or $w_{G}(u, v)$ the weight of the edge $e=(u, v) \in E(G)$. We also define $w(G)=$ $\sum_{e \in E(G)} w(e)$. Given an edge $e=(u, v)$, we denote by $G-e$ or $G-(u, v)$ (resp., $G+e$ or $G+(u, v))$ the graph obtained from $G$ by removing (resp., adding) the edge $e$. Similarly, for a set $F$ of edges, $G-F$ (resp., $G+F$ ) will denote the graph obtained from $G$ by removing (resp., adding) the edges in $F$.

We will call $\pi_{G}(x, y)$ a shortest path between two vertices $x, y \in V(G), d_{G}(x, y)$ its (weighted) length, and $T_{G}(s)$ a SPT of $G$ rooted at $s$. Whenever the graph $G$ and/or the vertex $s$ are clear from the context, we might omit them, i.e., we will write $\pi(u)$ and $d(u)$ instead of $\pi_{G}(s, u)$ and $d_{G}(s, u)$, respectively. When considering 
an edge $(x, y)$ of an SPT we will assume $x$ and $y$ to be the closest and the furthest endpoints from $s$, respectively.

Given an edge $e \in E(G)$, we define $\pi_{G}^{-e}(x, y), d_{G}^{-e}(x, y)$ and $T_{G}^{-e}(s)$ to be, respectively, a shortest path between $x$ and $y$, its length, and a SPT in the graph $G-e$. Moreover, if $P$ is a path from $x$ to $y$ and $Q$ is a path from $y$ to $z$, with $x, y, z \in$ $V(G)$, we will denote by $P \circ Q$ the path from $x$ to $z$ obtained by concatenating $P$ and $Q$.

Given $G$, a vertex $s \in V(G)$, and an edge $e=(u, v) \in E\left(T_{G}(s)\right)$, we denote by $U_{G}(e)$ and $D_{G}(e)$ the partition of $V(G)$ induced by the two connected components of $T(G)-e$, such that $U_{G}(e)$ contains $s$ and $u$, and $D_{G}(e)$ contains $v$. Then, $C_{G}(e)=$ $\left\{(x, y) \in E(G): x \in U_{G}(e), y \in D_{G}(e)\right\}$ will denote the cutset of $e$, i.e., the set of edges crossing the cut $\left(U_{G}(e), D_{G}(e)\right)$.

For the sake of simplicity we consider only edge weights that are strictly positive. However our entire analysis also extends to non-negative weights. We also assume, w.l.o.g., that the input graph $G$ is 2-edge/vertex-connected, to avoid pathological failures that would disconnect the graph. Throughout the rest of the paper we will assume that, when multiple shortest paths exist, ties will be broken in a consistent manner. In particular we fix a SPT $T=T_{G}(s)$ of $G$ and, given a graph $H \subseteq G$ and $x, y \in V(H)$, whenever we compute the path $\pi_{H}(x, y)$ and ties arise, we will prefer the edges in $E(T) 4$ We will also assume that if we are considering a shortest path $\pi_{H}(x, y)$ between $x$ and $y$ passing through vertices $x^{\prime}$ and $y^{\prime}$, then $\pi_{H}\left(x^{\prime}, y^{\prime}\right) \subseteq \pi_{H}(x, y)$.

\section{A 3-EASPT structure with at most $2 n$ edges}

We here provide a revisitation of one of the swap procedures presented in [20] to formally prove that it can be used to build a simple 3-EASPT with at most $2 n$ edges, on which our construction of the $(1+\varepsilon)$-EASPT will rely. More precisely, in $[20$, the authors were concerned with the problem of reconnecting in a best possible way (w.r.t. a set of distance criteria) the two subtrees of an SPT undergoing an edge failure, through a careful selection of a swap edge, i.e., an edge with an endvertex in each of the two subtrees. In particular, they show that if we select as a swap edge for $e=(u, v)$ - with $u$ closer to the source $s$ than $v$ - the edge that lies on a shortest path in $G-e$ from $s$ to $v$, then the distances from the source towards all the disconnected vertices is stretched at most by a factor of 35 Therefore, a 3 -EASPT of size at most $2 n$ can be obtained by simply adding to a SPT rooted at $s$ such a swap edge for each corresponding tree edge, and interestingly this improves the 3 -EASPT of size at most $4 n$ provided in [25.

More formally, Algorithm 1 builds a 3-EASPT $H$ as follows: initially $H$ is a shortest path tree of $G$ then, for each possible failure of an edge $e=(u, v)$ in $T_{G}(s)$, we augment $H$ by adding the (unique) edge $(x, y)$ of $C_{G}(e)$ that lies on a shortest path $\pi_{G}^{-e}(v)$ from $s$ to $v$. Notice that this is the only edge of $\pi_{G}^{-e}(v)$ that is not already in $T$ as both $\pi_{T}(s, x)$ and $\pi_{T}(v, y)$ do not contain $e$.

Lemma 1 Algorithm 1 computes in polynomial time a 3-EASPT structure of size $2 n-2$.

4 The notation $H \subseteq G$ means that $H$ is a subgraph of $G$.

5 Actually, in [20] it is not explicitly claimed the 3-stretch factor, but this is implicitly obtained by the qualitative analysis of the swap procedure therein provided. 


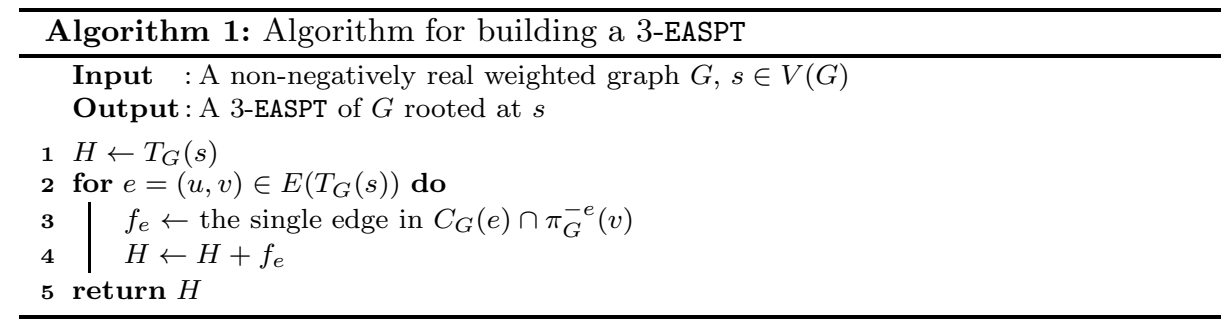

Proof The claim on the size of $H$ is a direct consequence of the fact that we add at most one replacement edge for each failure, so we only need to prove that $H$ is a 3-EASPT structure. As $H$ contains all the edges of $T_{G}(s)$ the condition $d_{H}(u)=d_{G}(u) \forall u \in V(G)$ is clearly true. Moreover, the above still holds whenever an edge $e \notin E\left(T_{G}(s)\right)$ fails.

Now, let $e=(u, v) \in E\left(T_{G}(s)\right)$ be the failed edge and let $t$ be any vertex in $V(G)$. If $t$ belongs to $U_{G}(e)$ then $H$ contains the whole shortest path $\pi_{G}^{-e}(t)=\pi_{G}(t)$. Otherwise, $H$ contains both the path $\pi_{G}^{-e}(v)$ and the path $\pi_{G}^{-e}(v, t)=\pi_{G}(v, t)$ so we can write:

$$
d_{H}^{-e}(t) \leq d_{G}^{-e}(v)+d_{G}(v, t) \leq d_{G}^{-e}(t)+2 d_{G}(v, t) \leq d_{G}^{-e}(t)+2 d_{G}(t) \leq 3 d_{G}^{-e}(t) .
$$

First, we give a high-level description of our algorithm for computing a $(1+\varepsilon)$ EASPT (see Algorithm 2). We build our structure $H$ by starting from the 3 -EASPT of size $O(n)$ returned by Algorithm 1 Then, our algorithm works in $n-1$ phases, where each phase considers the failure of an edge of $T$ w.r.t. a fixed preorder visit of the edges, say $e_{1}, \ldots, e_{n-1}$. Let $e=e_{h}$ be the edge of $T$ of the $h$-th phase of the algorithm. The algorithm checks all the vertices of $G$ in preorder w.r.t. $T_{G}^{-e}(s)$. Whenever a vertex $t$ is bad for e, i.e., $d_{H}^{-e}(t)>(1+\varepsilon) d^{-e}(t)$, the algorithm chooses a suitable value $\ell \geq 1$ and adds to $H$ all the last $\ell$ edges of $\pi^{-e}(t)$ that are missing. Notice that all the bad vertices for $e$ must necessarily belong to $D_{G}(e)$. As we will see, the choice of $\ell$ is done so that we do not only guarantee that $d_{H}^{-e}(t) \leq(1+\varepsilon) d^{-e}(t)$, but we also obtain a substantial improvement on the stretch factors of the distances from $s$ in $H-e$ for all the first $\ell-1$ predecessors of $t$ in $\pi^{-e}(t)$, say $t_{1}, \ldots, t_{\ell-1}$. Furthermore, as we will prove later, the structure $H$ built by the algorithm after that edge $e$ has been considered guarantees the following property: For every $h<i \leq n-1$, and for every $x \in\{t\} \cup\left\{t_{1}, \ldots, t_{\ell-1}\right\}$, we have that $d_{H}^{-e_{i}}(x) \leq d_{H}^{-e}(x)$. These are exactly the two key ingredients for the analysis of our algorithm that, combined altogether, allow us to prove that each vertex causes the addition of $O\left(\varepsilon^{-2} \log n\right)$ edges to $H$ on the average.

The main result we are going to prove in this section is the following:

Theorem 1 Given an n-vertex non-negatively real weighted graph $G$, a source vertex $s \in V(G)$, and any $\varepsilon>0$, the structure $H$ returned in polynomial time by Algorithm Q is a $(1+\varepsilon)$-EASPT of $G$ rooted at $s$ of size $O\left(\frac{n \log n}{\varepsilon^{2}}\right)$.

We will prove separately the bound on the stretch factor and on the size of the structure in the next two subsections (see Lemmas 2 and 9, respectively). 


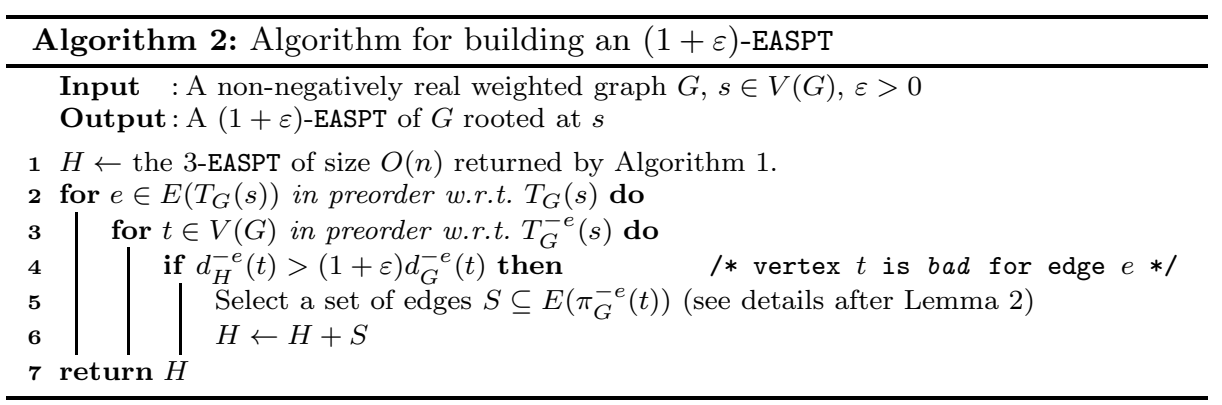

3.1 Stretch factor of the structure

Observe that, for every bad vertex $t$ for $e$, the algorithm always adds the last edge of $\pi^{-e}(t)$ to $H$. This is enough to prove the correctness of our algorithm as the following lemma shows.

Lemma 2 The structure $H$ returned by Algorithm 2 is a $(1+\varepsilon)$-EASPT.

Proof Let $\widetilde{H}$ be the structure built by the algorithm just before a bad vertex $t$ for an edge $e$ of $T$ is considered. Assume by induction that, for every vertex $x$ which has been visited by the algorithm before $t$ in the phase associated with $e$, we have $d_{\widetilde{H}}^{-e}(x) \leq(1+\varepsilon) d^{-e}(x)$. Let $f=\left(t^{\prime}, t\right)$ be the last edge of $\pi^{-e}(t)$. By induction, $d_{\widetilde{H}}^{-e}\left(t^{\prime}\right) \leq(1+\varepsilon) d^{-e}\left(t^{\prime}\right)$. Furthermore, as $t$ is bad for $e$, the algorithm adds $f$ to $\widetilde{H}$. Since $\widetilde{H}+f$ is a subgraph of $H$, we have that

$$
\begin{aligned}
d_{H}^{-e}(t) \leq d_{\widetilde{H}+f}^{-e}(t) \leq d_{\widetilde{H}}^{-e}\left(t^{\prime}\right)+w & (f) \leq(1+\varepsilon) d^{-e}\left(t^{\prime}\right)+w(f) \\
& \leq(1+\varepsilon)\left(d^{-e}\left(t^{\prime}\right)+d^{-e}\left(t^{\prime}, t\right)\right)=(1+\varepsilon) d^{-e}(t) .
\end{aligned}
$$

\section{A $(1+\varepsilon)$-EASPT structure}

\subsection{Size of the structure}

Now we describe the edge selection process and we analyze the size of our final structure. Let $H_{0}$ be the initial 3-EASPT structure. Let us fix the failed edge $e=$ $(u, v)$ and a single bad vertex $t$ for $e$. We call $H^{\prime}$ the structure built by the algorithm just before $t$ is considered. Let $f=(x, y)$ with $x \in U_{G}(e)$ be the unique edge in $C_{G}(e) \cap E\left(\pi_{G}^{-e}(t)\right)$. Consider the subpath of $\pi_{G}^{-e}(t)$ going from $x$ to $t$ and let $x_{0}, x_{1}, \ldots, x_{r}$ be its vertices, in order. We consider the set $Z=\left\{x_{i}:\left(x_{i-1}, x_{i}\right) \notin\right.$ $\left.E\left(H^{\prime}\right)\right\}$, we name its vertices $z_{1}, \ldots, z_{k}=t$ with $k=|Z|$, in order and we let $z_{0}=x$ (see Figure 21). We define $\alpha_{i}=\frac{d_{H^{\prime}}^{-e}\left(z_{i}\right)}{d^{-e}\left(z_{i}\right)}$. It follows from the definitions and from the proof of Lemma 2 that we have $\alpha_{0}=1, \alpha_{j} \leq(1+\varepsilon)$ for $1 \leq j<k$ and $\alpha_{k}>1+\varepsilon$.

Think of the edges in $\pi^{-e}(t)$ as being directed towards $t$ for a moment. In the following we will describe how to select the set $S$ of edges used by the algorithm. 


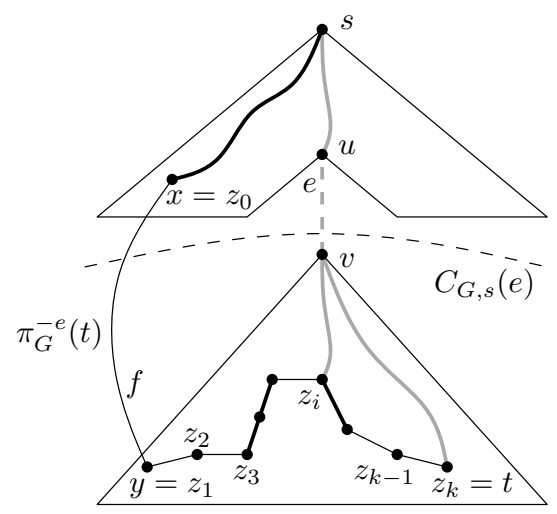

Fig. 2 Edge selection phase of Algorithm 2 when a bad vertex $t$ for the failing edge $e$ is considered. Bold edges belong to $H^{\prime}$ while the black path is $\pi_{G}^{-e}(t)$.

In particular, we will select $\eta \geq 1$ edges entering into the last $\eta$ vertices in $Z$. This choice of $S$ will ensure that the overall decrease of the values $\alpha_{i}$ in $H^{\prime}+S$ will be at least $\frac{\varepsilon}{\mathcal{H}_{n}} \eta$ where $\mathcal{H}_{n}$ denotes the $n$-th harmonic number. In particular, we exploit the fact that, after adding the set $S$, each "new value" $\alpha_{i}$ with $i>k-\eta$, will not be larger than $\alpha_{k-\eta}$ as we will show in the following.

Consider the sequence $\gamma_{0}, \ldots, \gamma_{k}$ where $\gamma_{i}=1+\frac{\varepsilon}{\mathcal{H}_{k}}\left(\mathcal{H}_{k}-\mathcal{H}_{k-i}\right)$. Notice that the sequence is monotonically increasing from $\gamma_{0}=1$ to $\gamma_{k}=1+\varepsilon$. Let $0 \leq j<k$ be the largest index such that $\alpha_{j} \leq \gamma_{j}$. Notice that $j$ always exists as $\alpha_{0}=\gamma_{0}$ and $\alpha_{k}>\gamma_{k}$. We set $\eta=k-j$ so that the set $S$ is defined accordingly. Let $U=\left\{z_{j+1}, \ldots, z_{k}\right\}$ be the set of vertices for which an incoming edges has been added in $S$.

For every vertex $z \in U$ we define the following path in $H^{\prime}+S-e: P(z)=$ $\pi_{H^{\prime}}^{-e}\left(z_{j}\right) \circ \pi\left(z_{j}, z\right)$. Notice that $\pi\left(z_{j}, z\right)$ is entirely contained in $H^{\prime}+S-e$. We define $\alpha_{i}^{\prime}=\frac{w\left(P\left(z_{i}\right)\right)}{d^{-e}\left(z_{i}\right)}$, and note that $\alpha_{i}^{\prime}$ is an upper bound to the stretch of $z$ in $H^{\prime}+S-e$.

Lemma 3 For $i>j, \alpha_{i}^{\prime} \leq \alpha_{j}<\alpha_{i}$.

Proof By definition of $j$, we have $\alpha_{j} \leq \gamma_{j}<\gamma_{i}<\alpha_{i}$. Now we prove $\alpha_{i}^{\prime} \leq \alpha_{j}$ :

$$
\alpha_{i}^{\prime}=\frac{w(P(z))}{d^{-e}\left(z_{i}\right)}=\frac{d_{H^{\prime}}^{-e}\left(z_{j}\right)+d\left(z_{j}, z_{i}\right)}{d^{-e}\left(z_{i}\right)} \leq \frac{\alpha_{j} d^{-e}\left(z_{j}\right)+d^{-e}\left(z_{j}, z_{i}\right)}{d^{-e}\left(z_{i}\right)} \leq \frac{\alpha_{j} d^{-e}\left(z_{i}\right)}{d^{-e}\left(z_{i}\right)}=\alpha_{j} .
$$

We now lower-bound the overall decrease of the values $\alpha_{i}^{\prime}$ 's w.r.t. the corresponding $\alpha_{i}$ 's by using the following inequalities (see Figure 3):

$$
\begin{aligned}
\sum_{z \in U}\left(\frac{d_{H^{\prime}}^{-e}(z)}{d^{-e}(z)}-\frac{w(P(z))}{d^{-e}(z)}\right) & =\sum_{i=j+1}^{k}\left(\alpha_{i}-\alpha_{i}^{\prime}\right) \geq \sum_{i=j+1}^{k}\left(\alpha_{i}-\alpha_{j}\right) \geq \sum_{i=j+1}^{k}\left(\gamma_{i}-\gamma_{j}\right) \\
& =\frac{\varepsilon}{\mathcal{H}_{k}} \sum_{i=j+1}^{k}\left(\mathcal{H}_{k-j}-\mathcal{H}_{k-i}\right)=\frac{\varepsilon}{\mathcal{H}_{k}}(k-j) \geq \frac{\varepsilon}{\mathcal{H}_{n}} \eta
\end{aligned}
$$




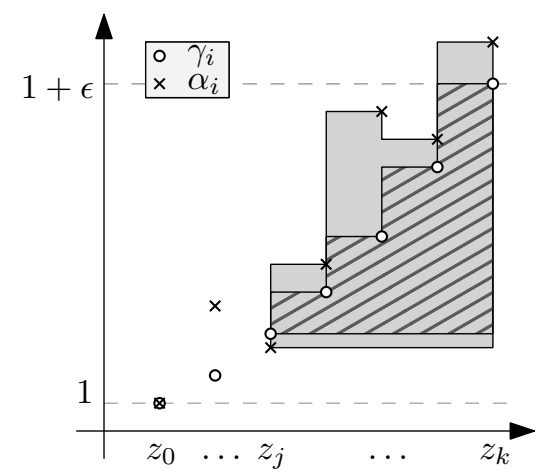

Fig. 3 Representation of the sequences $\alpha_{i}$ and $\gamma_{i}$. The gray area is a lower bound to the overall decrease of the $\alpha_{i}$ values w.r.t. $\alpha_{i}^{\prime}$ with $i>j$. This area is, in turn, lower bounded by the area of the striped region which is $\frac{\varepsilon}{\mathcal{H}_{k}}(k-j)$.

where in the last but one step we used the well-known equality that for every $j \leq k$, $\sum_{i=j+1}^{k}\left(\mathcal{H}_{k-j}-\mathcal{H}_{k-i}\right)=k-j$.

The above selection procedure is repeated by the algorithm for every failed edge $e_{h}$ and for every corresponding bad vertex. We now focus on the $h$-th phase of the algorithm. We call $B_{h}$ the set of all the bad vertices considered in this phase and, for every $t \in B_{h}$, we call $U(t)$ the corresponding set $U$, as defined above. Moreover, let $U_{h}=\cup_{t \in B} U(t)$, and let $V_{h}=\bigcup_{i=1}^{h} U_{i}$ (notice that $V_{0}=\emptyset$ ). Notice that the sets $U(t)$ are pairwise disjoint since, once $z \in U(t)$ we add the edge of $\pi^{-e}(t)$ entering $z$ and hence $z$ cannot belong to any other set $U\left(t^{\prime}\right)$ where $t^{\prime}$ is a bad vertex which is considered after $t$ in phase $h$. Hence, we let $P_{h}(z)$ be the unique path $P(z)$ which is built during phase $h$. Finally, let $H_{h}^{\prime}, H(t)$, and $H_{h}$ be the structures built by the algorithm at the beginning of phase $h$, just before the bad vertex $t \in B_{h}$ is processed, and at the end of phase of phase $h$, respectively.

Let $m_{h}$ be the number of new edges added during the phase $h$. We can now prove:

Lemma $4 \sum_{z \in U_{h}}\left(\frac{d_{H_{h}^{\prime}}^{-e_{h}}(z)}{d^{-e_{h}}(z)}-\frac{w\left(P_{h}(z)\right)}{d^{-e_{h}}(z)}\right) \geq m_{h} \frac{\varepsilon}{\mathcal{H}_{n}}$.

Proof For a bad vertex $t \in B_{h}$, let $\eta_{t}$ be the number of edges selected by the algorithm, i.e. $|S|$, when $t$ is considered. By summing Equation 1 over all vertices $t \in B_{h}$, we obtain:

$$
\begin{aligned}
\sum_{z \in U_{h}}\left(\frac{d_{H_{h}^{\prime}}^{-e_{h}}(z)}{d^{-e_{h}}(z)}\right. & \left.-\frac{w\left(P_{h}(z)\right)}{d^{-e_{h}}(z)}\right)=\sum_{t \in B_{h}} \sum_{z \in U(t)}\left(\frac{d_{H_{h}^{\prime}}^{-e_{h}}(z)}{d^{-e_{h}}(z)}-\frac{w\left(P_{h}(z)\right)}{d^{-e_{h}}(z)}\right) \\
& \geq \sum_{t \in B_{h}} \sum_{z \in U(t)}\left(\frac{d_{H(t)}^{-e_{h}}(z)}{d^{-e_{h}}(z)}-\frac{w\left(P_{h}(z)\right)}{d^{-e_{h}}(z)}\right) \geq \sum_{t \in B_{h}} \eta_{t} \frac{\varepsilon}{\mathcal{H}_{n}} \geq m_{h} \frac{\varepsilon}{\mathcal{H}_{n}}
\end{aligned}
$$

where we used the facts that the sets $U(t)$ are pairwise disjoint, and that every $H(t)$ is a supergraph of $H_{h}^{\prime}$. 
Now, let us define a function $\phi_{h}(z)$, first we set $\phi_{0}(z)=\frac{6}{\varepsilon} d(z)$ for every $z \in V$, and then we recursively define:

$$
\phi_{h}(z)= \begin{cases}w\left(P_{h}(z)\right) & \text { if } z \in U_{h} \\ \phi_{h-1}(z) & \text { if } z \notin U_{h}\end{cases}
$$

We will show that if $z \in V_{h}$, then $\phi_{h-1}(z)$ is an upper bound to $d_{H_{h}^{\prime}}^{-e_{h}}(z)$. In order to do so we separately consider the cases $z \in U_{h} \backslash V_{h-1}$ and $z \in V_{h-1}$ in the following two lemmas:

Lemma 5 For every $z \in U_{h} \backslash V_{h-1}$ we have $\phi_{h-1}(z) \geq d_{H_{h}^{\prime}}^{-e_{h}}(z)$.

Proof Since $z \in U_{h}$ we know that an incoming edge to $z$ has been selected when the algorithm was considering some bad vertex $t$ for the edge $e_{h}$. We have:

$$
\begin{aligned}
d^{-e_{h}}(v)+d(v, t)>(1+\varepsilon) d^{-e_{h}}(t)=(1+\varepsilon) & \left(d^{-e_{h}}(z)+d(z, t)\right) \\
& \geq(1+\varepsilon)\left(d^{-e_{h}}(z)+|d(z)-d(t)|\right) .
\end{aligned}
$$

Moreover, we also have:

$$
d^{-e_{h}}(v)+d(v, t) \leq d^{-e_{h}}(z)+d(z, v)+d(v, t) \leq d^{-e_{h}}(z)+d(z)+d(t)
$$

The above inequalities together imply:

$$
d^{-e_{h}}(z)<\frac{d(z)+d(t)-(1+\varepsilon)|d(z)-d(t)|}{\varepsilon} .
$$

If $d(z) \geq d(t)$, the above formula becomes:

$$
d^{-e_{h}}(z)<\frac{d(z)+d(t)-(1+\varepsilon)(d(z)-d(t))}{\varepsilon}=\frac{(2+\varepsilon) d(t)-\varepsilon d(z)}{\varepsilon} \leq \frac{2 d(z)}{\varepsilon} .
$$

Otherwise, $d(z)<d(t)$ and we have:

$$
d^{-e_{h}}(z)<\frac{d(z)+d(t)-(1+\varepsilon)(d(t)-d(z))}{\varepsilon}=\frac{(2+\varepsilon) d(z)-\varepsilon d(t)}{\varepsilon} \leq \frac{2 d(z)}{\varepsilon} .
$$

As $H_{h}$ is a supergraph of $H_{0}$, which is a 3-EASPT, we immediately have:

$$
d_{H_{h}^{\prime}}^{-e_{h}}(z) \leq d_{H_{0}}^{-e_{h}}(z) \leq 3 d^{-e_{h}}(z)<\frac{6}{\varepsilon} d(z)=\phi_{h-1}(z) .
$$

We now consider the remaining case:

Lemma 6 For $z \in V_{h-1}, \phi_{h-1}(z) \geq d_{H_{h}^{\prime}}^{-e_{h}}(z)$. 
Proof We show that the weight of every path $P_{h}(z)$ built by the algorithm when $e_{h}=(u, v)$ fails is an upper bound to $d_{H_{h^{\prime}}^{\prime}}^{-e_{h^{\prime}}}(z)$ for every $h^{\prime}>h$. This will immediately imply the claim. To prove the above we argue that $P_{h}(z)$ is vertex disjoint from $\pi^{-e_{h}}(v, z)$ (except for $z$ ). As a consequence, when $e_{h^{\prime}}$ fails either $P_{h}(z)$ is still in $H_{h^{\prime}}^{\prime}-e_{h^{\prime}}$ or $e_{h^{\prime}}$ is not in $\pi(z)$, hence $d_{H_{h^{\prime}}^{\prime}}^{-e_{h^{\prime}}}(z)=d(z)$.

Let $H^{\prime}$ be the structure constructed by the algorithm just before $P_{h}(z)$ is built, $t$ be the corresponding bad vertex, and $z_{j}$ be the vertex chosen as described above. Recall that $z=z_{i}$ for some $i>j$, and that $P_{h}(z)=\pi_{H^{\prime}}^{-e_{h}}\left(z_{j}\right) \circ \pi\left(z_{j}, z\right)$. Suppose by contradiction that $P_{h}(z)$ and $\pi(v, z)$ intersect at some vertex $q \neq z$. Clearly $q \in D(e)$. If $q \in V\left(\pi\left(z_{j}, z\right)\right)$ then $\pi\left(z_{j}, z\right)$ contains $\pi(q, z)$ as a subpath 6 As $\pi\left(z_{j}, z\right)$ is, in turn, a subpath of $\pi^{-e_{h}}(z)$, this implies that the edge preceding $z$ in $\pi^{-e_{h}}(z)$ belongs to $T \subseteq H_{0}$ and this contradicts the definition of $z$.

Otherwise $q \in V\left(\pi_{H^{\prime}}^{-e_{h}}\left(z_{j}\right)\right)$. As $z_{j}$ precedes $z$ in $\pi^{-e}(t)$ we have $d^{-e}\left(z_{j}\right) \leq$ $d^{-e}(z)$. Since $\pi^{-e}(q, z)=\pi(q, z)$ which is in $H^{\prime}$, we can write:

$$
\begin{aligned}
\alpha_{i}=\frac{d_{H^{\prime}}^{-e}(z)}{d^{-e}(z)} \leq \frac{d_{H^{\prime}}^{-e}(q)+d^{-e}(q, z)}{d^{-e}(z)} \leq \frac{d_{H^{\prime}}^{-e}(q)+d^{-e}\left(q, z_{j}\right)+d^{-e}\left(z_{j}, z\right)}{d^{-e}(z)}= \\
\quad \frac{d_{H^{\prime}}^{-e}\left(z_{j}\right)+d^{-e}\left(z_{j}, z\right)}{d^{-e}\left(z_{j}\right)+d^{-e}\left(z_{j}, z\right)} \leq \max \left\{\frac{d_{H^{\prime}}^{-e}\left(z_{j}\right)}{d^{-e}\left(z_{j}\right)}, \frac{d^{-e}\left(z_{j}, z\right)}{d^{-e}\left(z_{j}, z\right)}\right\}=\max \left\{\alpha_{j}, 1\right\}=\alpha_{j}
\end{aligned}
$$

where we used that for every $a, b, c, d>0$, we have that $\frac{a+b}{c+d} \leq \max \left\{\frac{a}{c}, \frac{b}{d}\right\}$, and the inequality $\alpha_{j} \geq 1$. The above contradicts Lemma 3 .

To summarize, combining Lemma 5 and 6 together, we immediately have:

Corollary 1 If $z \in V_{h}$, then $\phi_{h-1}(z) \geq d_{H_{h}^{\prime}}^{-e_{h}}(z)$.

Next lemma shows that $\phi_{h}(z)$ is monotonically non-increasing w.r.t. $h$ :

Lemma 7 For every $h \geq 1, \phi_{h-1}(z) \geq \phi_{h}(z)$.

Proof If $z \notin U_{h}$ then, by definition, we have $\phi_{h-1}(z)=\phi_{h}(z)$. Otherwise $z \in U_{h}$, let $\widetilde{H}$ be the structure constructed by the algorithm just before $P_{h}(z)$ is built and recall that $z=z_{i}$ for some $i$. As $z \in U_{h}$ we have $H_{h}^{\prime} \subseteq \widetilde{H}$, moreover Corollary 1 holds, hence we can write $\phi_{h-1}(z) \geq d_{H_{h}^{\prime}}^{-e_{h}}(z) \geq d_{\widetilde{H}}^{-e_{h}}(z)$. The claim follows as we have:

$$
d_{\widetilde{H}}^{-e_{h}}(z) \geq \phi_{h}(z) \Longleftrightarrow \frac{d_{\widetilde{H}}^{-e_{h}}(z)}{d^{-e_{h}}(z)} \geq \frac{w\left(P_{h}(z)\right)}{d^{-e_{h}}(z)} \Longleftrightarrow \alpha_{i} \geq \alpha_{i}^{\prime}
$$

which is true by Lemma 3 .

We now define a non-increasing global potential function $\Phi(h)$ for $0<h \leq n-1$ :

$$
\Phi(h)=\sum_{z \in V \backslash\{s\}} \frac{\phi_{h}(z)}{d(z)} .
$$

The following lemma bounds the decrease of $\Phi$ after each phase of the algorithm:

\footnotetext{
6 This is due to the tie-breaking rule discussed before which gives priority to edges in $T$.
} 
Lemma $8 \Phi(h-1)-\Phi(h) \geq m_{h} \frac{\varepsilon}{\mathcal{H}_{n}}$.

Proof Using the definitions we have:

$$
\begin{aligned}
& \Phi(h-1)-\Phi(h)=\sum_{z \in V \backslash\{s\}} \frac{\phi_{h-1}(z)}{d(z)}-\sum_{z \in V \backslash\{s\}} \frac{\phi_{h}(z)}{d(z)}= \\
& \sum_{z \in U_{h}} \frac{\phi_{h-1}(z)-\phi_{h}(z)}{d(z)}+\sum_{z \in V \backslash\left(U_{h} \cup\{s\}\right)} \frac{\phi_{h-1}(z)-\phi_{h}(z)}{d(z)}=\sum_{z \in U_{h}} \frac{\phi_{h-1}(z)-\phi_{h}(z)}{d(z)}
\end{aligned}
$$

where the latter equality follows from the fact that $\phi_{h}(z)=\phi_{h-1}(z)$ whenever $z \notin$ $U_{h} \cup\{s\}$. Starting from the latter quantity, we use Lemma 7 and then Corollary 1 to write:

$$
\sum_{z \in U_{h}} \frac{\phi_{h-1}(z)-\phi_{h}(z)}{d(z)} \geq \sum_{z \in U_{h}} \frac{\phi_{h-1}(z)-\phi_{h}(z)}{d^{-e_{h}}(z)} \geq \sum_{z \in U_{h}}\left(\frac{d_{H_{h}^{\prime}}(z)}{d^{-e_{h}}(z)}-\frac{w\left(P_{h}(z)\right)}{d^{-e_{h}}(z)}\right) .
$$

which is at least $m_{h} \frac{\varepsilon}{\mathcal{H}_{n}}$ as shown by Lemma 4 .

We are finally able to prove the following:

Lemma 9 The size of the structure $H$ returned by Algorithm 0 is $O\left(\frac{n \log n}{\varepsilon^{2}}\right)$.

Proof Since $H_{0}$ contains $O(n)$ edges, we only focus on bounding the number $\mu=$ $\sum_{h=1}^{n-1} m_{h}$ of edges in $E(H) \backslash E\left(H_{0}\right)$. Notice that, by definition of $\phi_{0}(z)$, we have $\Phi(0)=\sum_{z \in V \backslash\{s\}} \frac{\phi_{0}(z)}{d(z)} \leq \frac{6}{\varepsilon} n$. Moreover, as every $\phi_{h}(z)$ is non-negative, $\Phi(n-1) \geq$ 0 holds. Using these inequalities together with Lemma 8 , we can write:

$$
\frac{6}{\varepsilon} n \geq \Phi(0)-\Phi(n-1)=\sum_{h=1}^{n-1}(\Phi(h-1)-\Phi(h)) \geq \frac{\varepsilon}{\mathcal{H}_{n}} \sum_{h=1}^{n-1} m_{h}=\frac{\varepsilon}{\mathcal{H}_{n}} \mu
$$

which can be solved for $\mu$ to get $\mu=O\left(\frac{n \log n}{\varepsilon^{2}}\right)$.

\section{$5 \mathbf{A}(1+\varepsilon)$-VASPT structure}

In this section we extend our previous $(1+\varepsilon)$-EASPT structure to deal with vertex failures. In order to do so we build a different initial subgraph $H_{0}$, which is a 3 -VASPT having suitable properties that we will describe later. Then we use the natural extension of Algorithm 2 where we consider (in preorder) vertex failures instead of edge failures.

The construction of the subgraph $H_{0}$ is similar to that given by Baswana and Khanna [5] for the related problem of computing a vertex-fault-tolerant SDSO which reports (post-failure) 3-approximate distances from $s$. In particular, the key difference between their construction and ours is pointed out within the proof of the forthcoming Lemma 11, and such a difference is instrumental to guarantee the correctness of our approach. In the following, we first describe the construction of our structure $H_{0}$, and then we argue on how the analysis for the edge-failure case can be adjusted to show the same bound on the size of $H$ for the vertex failure case as well. 


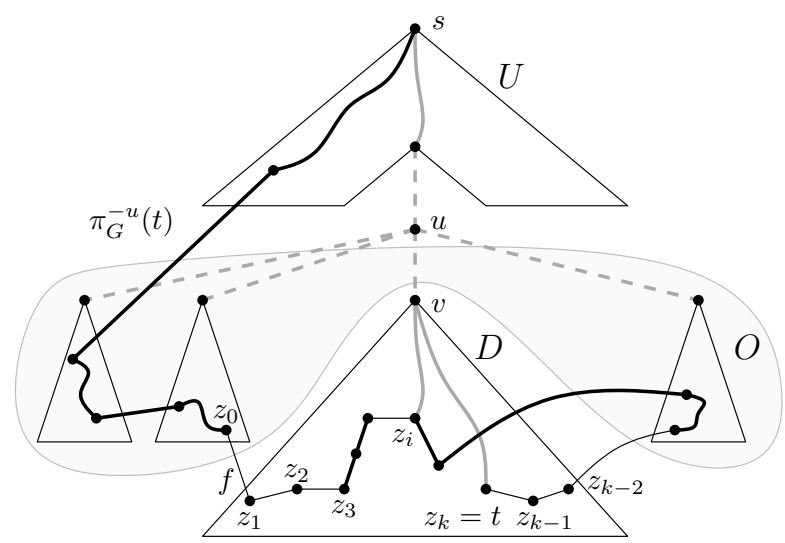

Fig. 4 Edge selection phase of the vertex-version of Algorithm 2 when a bad vertex $t$ for the failing vertex $u$ is considered. Bold edges belong to $H^{\prime}$ while the black path is $\pi_{G}^{-u}(t)$. Notice that all $z_{i}$ s belong to the down set $D$.

Initially, $H_{0}$ is equal to $T$. Then, proceeding as proposed in [5], $T$ is decomposed into ancestor-leaf vertex-disjoint paths in the following recursive way: select a path $Q$ from the root of $T$ to a leaf such that the removal of $Q$ splits the tree into a forest where the size of each subtree is at most half the size of the original tree, and then proceed recursively on each subtree. After this preliminary path-decomposition step of $T$, for each generated path an approximate structure is built. This structure will provide approximate distances towards the vertices $V(G) \backslash\{u\}$ whenever any vertex $u$ along the path fails. The union of $T$ with all these structures will form $H_{0}$.

Let us then describe how to build the initial structure for a fixed path $Q$ of the previous decomposition. Let $q$ be the starting vertex of $Q$, and let $T_{q}$ be the subtree of $T$ rooted at $q$. Moreover, let $u \in V(Q)$ be a failing vertex, and let $v$ be the next vertex in $Q \sqrt{7}$ Similarly to what is done in [5], we partition the vertices of the forest $T-u$ into three sets: (i) the up set $U$ containing all the vertices of the tree rooted at $s$, (ii) the down set $D$ containing all the vertices of the tree rooted at $v$, and (iii) the others set $O$ containing all the remaining vertices (see Figure 4).

In order to select the set of additional edges associated with $Q$, we construct a SPT $T^{\prime}$ of $G-u$ and we imagine that its edges are directed towards the leaves. We select all the edges of $E\left(T^{\prime}\right) \backslash E(T)$ that do not lead to a vertex in $D$, plus the unique edge of $\pi^{-u}(v)$ that crosses the cut induced by the sets $U \cup O$ and $D$. Notice that $T-u$ contains all the paths in $T^{\prime}$ towards the vertices in $U$, and that each vertex has at most one incoming edge in $T^{\prime}$. This implies that the number of selected edges is at most $|O|+1$.

The above procedure is repeated for all the failing vertices of $Q$, in order. As the sets $O$ associated with the different vertices are disjoint we have that, while processing $Q$, at most $\left|V\left(T_{q}\right)\right|+|Q|=O\left(\left|V\left(T_{q}\right)\right|\right)$ edges are selected. Finally, the procedure is repeated for all the paths of the decomposition, and since such a

7 W.l.o.g. we are assuming that the failing vertex $u$ is not a leaf, as otherwise $T-u$ is already a SPT of $G-u$. 
decomposition is done as suggested in [5], it immediately follows that the size of the entire structure $H_{0}$ is $O(n \log n)$.

We now prove some useful properties of the structure $H_{0}$. First of all, observe that, by construction and similarly to the edge-failure case, we immediately have:

Lemma 10 Let $u$ be a failed vertex and consider another vertex $z \neq u$. We have: (i) $d_{H_{0}}^{-u}(v)=d^{-u}(v)$, and (ii) for $z \in D$, it holds $d_{H_{0}}^{-u}(z) \leq 3 d^{-u}(z)$.

Moreover, we also have the following:

Lemma 11 Consider a failed vertex $u$. During the execution of the vertex-version of Algorithm 2, every bad vertex $t$ for $u$ will be in $D$.

Proof Let $\widetilde{H}$ be the structure build by the algorithm just before $t$ is considered. Assume by contradiction that $t \notin D$. Clearly, $t$ cannot be in $U$ so we must have $t \in O$. By construction of $H_{0}$, the path $\pi^{-u}(t)$ must contain some vertex of $D$. Let $z$ be the last vertex of $\pi^{-u}(t)$ that is also in $D$. As $z$ precedes $t$ in $\pi^{-u}(t)$ we must have $d_{\widetilde{H}}^{-u}(z) \leq(1+\varepsilon) d^{-u}(z)$. Moreover, by construction, $\pi^{-u}(z, t)$ is entirely contained in $H_{0} 8$ This implies:

$$
d_{\widetilde{H}}^{-u}(t) \leq d_{\widetilde{H}}^{-u}(z)+d^{-u}(z, t) \leq(1+\varepsilon) d^{-u}(z)+d^{-u}(z, t) \leq(1+\varepsilon) d^{-u}(t) .
$$

which contradicts the fact that $t$ is a bad vertex for $u$.

At this point, the same analysis given for the case of edge failures can be retraced for vertex failures as well. We point out that Lemma 11 ensures that every bad vertex for $u$ is in the same subtree as $v$. Also notice that all the vertices $z_{i}$ 's are, by definition, in the same subtree as well (see Figure (4). The above, combined with Lemma 10 (i), is needed by the proof of Lemma 5 , while Lemma 10 (ii) is used in the proof of Lemma 8 . Hence we have:

Theorem 2 Given an n-vertex non-negatively real weighted graph $G$, a source vertex $s \in V(G)$, and any $\varepsilon>0$, the vertex-version of Algorithm 2 computes in polynomial time $a(1+\varepsilon)$-VASPT of $G$ rooted at s of size $O\left(\frac{n \log n}{\varepsilon^{2}}\right)$.

\section{Relation with $(\alpha, \beta)$-spanners in unweighted graphs}

In this section we turn our attention to the unweighted case, and we provide two polynomial-time algorithms that augment an $(\alpha, \beta)$-spanner of $G$ so to obtain an $(\alpha, \beta)$-EABFS/VABFS. We present the algorithm for the vertex-failure case, and then we show how it can be adapted to the edge-failure case.

The algorithm first augments the structure $H_{0}$ computed as explained in Section 5. and then adds its edges to the $(\alpha, \beta)$-spanner of $G$. The structure $H_{0}$ is augmented as follows. The vertices of the BFS of $G$ rooted at $s$ are visited in preorder. Let $u$ be the vertex visited by the algorithm and let $D$ be the set of vertices of the tree defined so as explained in Section 5 w.r.t the path decomposition computed for $H_{0}$. For every $t \in D$, the algorithm checks whether $\pi_{G}^{-u}(s, t)$ contains no

8 Notice that this property would not be guaranteed by the initial structure provided in 5, and it is exactly the key difference between our construction and the one given in [5]. 
vertex of $D \backslash\{t\}$ and $d_{G}^{-u}(s, t)<d_{H_{0}}^{-u}(s, t)$. If this is the case, then the algorithm augments $H_{0}$ with the edge of $\pi_{G}^{-u}(s, t)$ incident to $t$.

The following observation is crucial to prove the algorithm correctness.

Fact 1 For every vertex $u$ and every vertex $t \in V(G) \backslash\{u\}$ such that $\pi_{G}^{-u}(t)$ contains a vertex in $D$, let $x$ and $y$ be the first and last vertex of $\pi_{G}^{-u}(t)$ that belong to $D$, respectively. We have $d_{H_{0}}^{-u}(x)=d_{G}^{-u}(x)$ and $d_{H_{0}}^{-u}(y, t)=d_{G}^{-u}(y, t)$.

We can now give the following:

Theorem 3 Given an unweighted graph $G$ with $n$ vertices and $m$ edges, a source vertex $s \in V(G)$, and an $(\alpha, \beta)$-spanner for $G$ of size $\sigma=\sigma(n, m)$, it can be computed in polynomial time an $(\alpha, \beta)-V A B F S$ w.r.t. $s$ of size $O(\sigma+n \log n)$.

Proof Let $H$ be the subgraph of $G$ computed by the algorithm. We first prove that $H$ is an $(\alpha, \beta)$-VABFS of $G$ and $s$ by showing that $d_{H}^{-u}(s, t) \leq \alpha \cdot d_{G}^{-u}(s, t)+\beta$, for two distinct vertices $u, t \in V(G)$. W.l.o.g., we can assume that $\pi_{G}^{-u}(s, t)$ contains some vertices of $D \backslash\{t\}$ because, if our assumption was not true, then, by Fact 1 . $d_{H}^{-u}(s, t)=d_{G}^{-u}(s, t) \leq \alpha \cdot d_{G}^{-u}(s, t)+\beta$.

Let $x$ and $y$ be the first and last vertex of $\pi_{G}^{-u}(s, t)$ contained in $D \backslash\{t\}$ in a path traversal from $s$ to $t$, respectively. We have that $\pi_{G}^{-u}(s, t)=\pi_{G}^{-u}(s, x) \circ$ $\pi_{G}^{-u}(x, y) \circ \pi_{G}^{-u}(y, t)$, i.e.,

$$
d_{G}^{-u}(s, t)=d_{G}^{-u}(s, x)+d_{G}^{-u}(x, y)+d_{G}^{-u}(y, t) .
$$

By Fact 1, $H$ contains $\pi_{G}^{-u}(s, x)$ as well as $\pi_{G}^{-u}(y, t)$. Therefore,

$$
d_{H}^{-u}(s, x)=d_{G}^{-u}(s, x) \quad \text { and } \quad d_{H}^{-u}(y, t)=d_{G}^{-u}(y, t) .
$$

We now prove that $d_{H}^{-u}(x, y) \leq \alpha \cdot d_{G}^{-u}(x, y)+\beta$. Since $H$ contains an $(\alpha, \beta)$-spanner of $G, H$ contains a path $P$ from $x$ to $y$ such that $w(P) \leq \alpha \cdot d_{G}(x, y)+\beta$. Clearly, if $u \notin V(P)$, then $H-u$ contains $P$ and therefore $d_{H}^{-u}(x, y) \leq w(P) \leq \alpha \cdot d_{G}(x, y)+\beta$. Otherwise, if $u \in V(P)$, then let $v$ be the least common ancestor of $x$ and $y$ in the BFS of $G$ rooted at $s$. Since $v \in D$, it follows that

$$
d_{H}^{-u}(x, y) \leq d_{H}(x, v)+d_{H}(v, y)<d_{H}(x, u)+d_{H}(u, y) \leq w(P) \leq \alpha \cdot d_{G}(x, y)+\beta .
$$

Using the last inequality together with Equations (2) and (3), we have that

$$
\begin{aligned}
d_{H}^{-u}(s, t) & \leq d_{H}^{-u}(s, x)+d_{H}^{-u}(x, y)+d_{H}^{-u}(y, t) \\
& \leq d_{G}^{-u}(s, x)+\alpha \cdot d_{G}(x, y)+\beta+d_{G}^{-u}(y, t) \leq \alpha \cdot d_{G}^{-u}(s, t)+\beta .
\end{aligned}
$$

We now prove that the size of $H$ is $O(\sigma+n \log n)$ by showing that the size of $H_{0}$ is $O(n \log n)$. We have already shown in the previous section that the number of edges of $H_{0}$ before the algorithm augments it is $O(n \log n)$. Therefore, it remains to bound the number of edges added to $H_{0}$. Let $F$ be the set of such edges. We prove that $|F| \leq 3 n$ by showing that each vertex $t$ caused the addition of at most 3 edges to $F$. Let $t$ be a fixed vertex. Let $u_{0}, \ldots, u_{\ell}$ be the vertices of the path $\pi_{G}(s, t)$, in a traversal of the path from $s$ to $t$ whose failures caused the insertion of the edge $\left(v_{i}, t\right)$ of $\pi_{G}^{u_{i}}(s, t)$ incident to $t$ in $F$. Since $G$ is unweighted, $d_{G}(s, t)=d_{G}\left(s, v_{i}\right)+j$, where $j \in\{-1,0,1\}$. Furthermore, for every vertex $u^{\prime} \neq t$ which is a proper descendent of $u_{0}$ in the BFS tree of $G$ rooted at $s, H-u^{\prime}$ contains the path $\pi_{G}\left(s, v_{0}\right) \circ \pi_{G}\left(v_{0}, t\right)$ 
of length at most $d_{G}(s, t)+1+1=d_{G}(s, t)+2$. Finally, observe that for every $1 \leq i \leq \ell$ and for every vertex $u^{\prime} \neq t$ which is a descendent of $u_{i}$ in the BFS tree of $G$ rooted at $s, H-u^{\prime}$ contains the path $\pi_{G}^{-u_{i}}(s, t)$. Therefore, for every $2 \leq i \leq \ell$, we have that

$$
d_{G}(s, t) \leq d_{G}^{-u_{i}}(s, t) \leq d_{G}(s, t)+2-i .
$$

The above inequality implies that $\ell \leq 2$. Hence each vertex $t$ caused the addition of at most $\ell+1 \leq 3$ edges to $F$.

Now, we adapt the algorithm to prove a similar result for the $(\alpha, \beta)$-EABFS. The algorithm first augments a BFS tree $T$ of $G$ rooted at $s$ and then adds its edges to the $(\alpha, \beta)$-spanner of $G$. The tree $T$ is augmented by visiting its edges in preorder. Let $e$ be the edge visited by the algorithm. For every $t \in D_{G}(e)$, the algorithm checks whether $\pi_{G}^{-e}(s, t)$ contains no vertex of $D_{G}(e) \backslash\{t\}$ and $d_{G}^{-e}(s, t)<d_{T}^{-e}(s, t)$. If this is the case, then the algorithm augments $T$ with the edge of $\pi_{G}^{-e}(s, t)$ incident to $t$. It is easy to see that the proof of Theorem 3 can be adapted to prove the following:

Theorem 4 Given an unweighted graph $G$ with $n$ vertices, a source vertex $s \in V(G)$, and an $(\alpha, \beta)$-spanner for $G$ of size $\sigma$, it can be computed in polynomial time an $(\alpha, \beta)$ EABFS w.r.t. $s$ of size less than or equal to $\sigma+3 n$.

Notice that the obtained $(\alpha, \beta)$-E/VABFS structures can be easily adapted to the multisource case, by simply rooting at each given source vertex $s \in S$ an augmented BFS. This will immediately provide corresponding $(\alpha, \beta)$-stretched sourcewise edge/vertex-fault-tolerant spanners (SES/SVS) of size $O(\sigma+|S| \cdot n)$ and $O(\sigma+|S| \cdot n \log n)$, respectively.

Interestingly, this immediately allows to improve some existing contructions. Indeed, by using the $(1,4)$-spanner of size $\widetilde{O}\left(n^{\frac{7}{5}}\right)$ given in [1] we obtain the following result:

Corollary 2 Given an unweighted graph $G$ with $n$ vertices, and a set of source vertices $S \subseteq V(G)$, we can compute in polynomial time a $(1,4)$-SES of $G$ w.r.t. $S$ having size $\widetilde{O}\left(n^{\frac{7}{5}}+|S| \cdot n\right)$.

This sparsifies the $(1,4)$-SES of size $O\left(|S| \cdot n^{\frac{4}{3}}\right)$ given in [25] as soon as $|S|=\widetilde{\omega}\left(n^{\frac{1}{15}}\right)$.

Moreover, by using the $(1,6)$-spanner of size $O\left(n^{4 / 3}\right)$ provided in [4, we also have:

Corollary 3 Given an unweighted graph $G$ with $n$ vertices, and a set of source vertices $S \subseteq V(G)$, we can compute in polynomial time a $(1,6)$-SVS of $G$ w.r.t. $S$ having size $O\left(n^{\frac{4}{3}}+|S| \cdot n \log n\right)$.

This improves the additive stretch of the $(1,8)$-SVS of size $\widetilde{O}\left(n^{\frac{4}{3}}\right)$ given in [21], which holds for $|S|=\widetilde{O}\left(n^{\frac{1}{3}}\right)$.

\section{Conclusions}

In this paper, we have studied the problem of designing single-edge/vertex-faulttolerant structures rooted at a source vertex, aiming at finding a compact set of 
edges of the input (either weighted or unweighted) graph that will provide approximate shortest paths from the source following the failure of an edge/vertex in the graph. The main contribution of our research is that we can get almost shortest paths with almost linear size, in sharp contrast with a corresponding true-shortest paths structure which may require a quadratic size. Another interesting contribution we provided is the bridging between $(\alpha, \beta)$-spanners and $(\alpha, \beta)$-E/VABFS.

The problem of designing good fault-tolerant approximate-shortest-path structures deserves further investigation. For the single-source case, we mention three intriguing problems: (1) designing a SDSO with stretch arbitrary close to 1, almost linear size and constant query time for both the single-edge and the single-vertex failure scenario. The closest result is the SDSO given in 8 that has a logarithmic query time (w.r.t. the number of vertices of the graph) and only works for single edge failures; (2) removing the log-factor from the size of our structure, either improving its analysis or by further sparsifying it; (3) studying the multiple vertexfailure case. To the best of our knowledge there are no non-trivial VASPTs or SDSOs for this case. Other future directions involve the study of the multisource case (i.e., a sourcewise fault-tolerant spanner), with the goal of designing a structure which only adds a sublinear (in the number of sources) term to the size of our singlesource structure. Moreover, we also plan to investigate the existence of efficient fault-tolerant structures for other notable network topologies, like the minimum spanning tree, the tree spanner, or the minimum-routing cost spanning tree.

\section{References}

1. I. Althöfer, G. Das, D.P. Dobkin, D. Joseph, and J. Soares, On sparse spanners of weighted graphs, Discrete \& Computational Geometry, 9:81-100, 1993.

2. G. Ausiello, A. Ribichini, P.G. Franciosa, and G.F. Italiano, Computing graph spanners in small memory: Fault-tolerance and streaming, Discrete Mathematics, Algorithms and Applications, 2(4):591-606, 2010.

3. G. Ausiello, P.G. Franciosa, G.F. Italiano, and A. Ribichini, On resilient graph spanners, Proc. of the 21st European Symp. on Algorithms (ESA'13), Vol. 8125 of Lecture Notes in Computer Science, Springer, 85-96, 2013.

4. S. Baswana, T. Kavitha, K. Mehlhorn, and S. Pettie, Additive spanners and $(\alpha, \beta)$ spanners, ACM Trans. on Algorithms, 7, A.5, 2010.

5. S. Baswana and N. Khanna, Approximate shortest paths avoiding a failed vertex: near optimal data structures for undirected unweighted graphs, Algorithmica, 66(1):18-50, 2013.

6. A. Bernstein and D.R. Karger, A nearly optimal oracle for avoiding failed vertices and edges, Proc. of the 41st Symp. on the Theory of Computing (STOC'09), ACM Press, 101-110, 2009.

7. D. Bilò, F. Grandoni, L. Gualà, S. Leucci, and G. Proietti, Improved purely additive faulttolerant spanners, Proc. of the 23rd European Symposium on Algorithms (ESA'15), Vol. 9294 of Lecture Notes in Computer Science, Springer, 167-178, 2015.

8. D. Bilò, L. Gualà, S. Leucci, and G. Proietti, Compact and fast sensitivity oracles for singlesource distances, Proc. of the 24th Annual European Symposium on Algorithms (ESA'16), Vol. 57 of Leibniz International Proceedings in Informatics (LIPIcs), 13:1-13:14, 2016.

9. D. Bilò, L. Gualà, S. Leucci, and G. Proietti, Multiple-edge-fault-tolerant approximate shortest-path trees, Proc. of the 33rd Symposium on Theoretical Aspects of Computer Science (STACS'16), Vol. 47 of Leibniz International Proceedings in Informatics (LIPIcs), 18:1-18:14, 2016.

10. G. Braunschvig, S. Chechik, and D. Peleg, Fault tolerant additive spanners, Proc. of the 38th Workshop on Graph-Theoretic Concepts in Computer Science (WG'12), Vol. 7551 of Lecture Notes in Computer Science, Springer, 206-214, 2012.

11. S. Chechik, New additive spanners, Proc. of the 24th Symp. on Discrete Algorithms (SODA'13), ACM Press, 498-512, 2013. 
12. S. Chechik, M. Langberg, D. Peleg, and L. Roditty, Fault-tolerant spanners for general graphs, Proc. of the 41st Symp. on the Theory of Computing (STOC'09), ACM Press, 435-444, 2009.

13. S. Chechik, M. Langberg, D. Peleg, and L. Roditty, $f$-sensitivity distance oracles and routing schemes, Proc. of the 18th European Symp. on Algorithms (ESA'10), Vol. 6942 of Lecture Notes in Computer Science, Springer, 84-96, 2010.

14. M. Cygan, F. Grandoni, and T. Kavitha, On pairwise spanners, Proc. of the Symp. on Theoretical Aspects of Computer Science (STACS'13), Vol. 20 of Leibniz International Proceedings in Informatics (LIPIcs), 209-220, 2013.

15. A. D'Andrea, M. D'Emidio, D. Frigioni, S. Leucci, and G. Proietti, Path-fault-tolerant approximate shortest-path trees, Proc. of the 22nd Int. Coll. on Structural Information and Communication Complexity (SIROCCO'15), Vol. 9439 of Lecture Notes in Computer Science, Springer, 224-238, 2015.

16. M. Dinitz and R. Krauthgamer, Fault-tolerant spanners: better and simpler, Proc. of the 30th Symp. on Principles of Distributed Computing (PODC'11), ACM Press, 169-178, 2011.

17. R. Duan and S. Pettie, Dual-failure distance and connectivity oracles, Proc. of the 20th Symp. on Discrete Algorithms (SODA'09), ACM Press, 506-515, 2009.

18. P. Erdős, Extremal problems in graph theory, Proc. of the Symp. on Theory of Graphs and its Applications, 29-36, 1964

19. F. Grandoni and V. Vassilevska Williams, Improved distance sensitivity oracles via fast single-source replacement paths, Proc. of the 53rd Annual IEEE Symp. on Foundations of Computer Science (FOCS'12), 748-757, 2012.

20. E. Nardelli, G. Proietti, and P. Widmayer, Swapping a failing edge of a single source shortest paths tree is good and fast, Algorithmica, 36(4):361-374, 2003.

21. M. Parter, Vertex fault tolerant additive spanners, Proc. of the 28th International Symposium on Distributed Computing (DISC'14), Vol. 8784 of Lecture Notes in Computer Science, Springer, 167-181, 2014.

22. M. Parter, Dual failure resilient BFS structure, Proc. of the 34th Symp. on Principles of Distributed Computing (PODC'15), ACM Press, 481-490, 2015.

23. M. Parter, Fault-Tolerant Logical Network Structures, Bulletin of the EATCS, Vol. 118, 2016.

24. M. Parter and D. Peleg, Sparse fault-tolerant BFS trees, Proc. of the 21st European Symp. on Algorithms (ESA'13), Vol. 8125 of Lecture Notes in Computer Science, Springer, 779790, 2013.

25. M. Parter and D. Peleg, Fault tolerant approximate BFS structures, Proc. of the 25th Symp. on Discrete Algorithms (SODA'14), ACM Press, 1073-1092, 2014. 